\title{
Variations plasmatiques postprandiales de 3-méthylhistidine comparée à celles de lysine, d'homoarginine et de xylose dans des conditions normales et en cas de malabsorption chez le veau pré-ruminant
}

\author{
PY Vacher 1, M Schmitz 2, H Hirni 1, JW Blum 1* \\ ${ }^{1}$ Inst für Tierzucht der Universität, Abt für Ernährungspathologie, 3012 Berne, Suisse; \\ 2 Bundesanstalt für Milchforschung, Inst für Physiologie \\ und Biochemie der Ernährung, 2300 Kiel 14, RFA
}

(Reçu le 27 novembre 1989; accepté le 20 mars 1990)

\begin{abstract}
Résumé - Nous avons étudié l'absorption de la 3-méthylhistidine (3MH) in vitro et les variations de la concentration plasmatique de $3 \mathrm{MH}$ chez des veaux pré-ruminants, après l'ingestion de lait contenant de la $3 \mathrm{MH}$, dans des situations normales et de malabsorption. Les variations ont été comparées à celles concernant la lysine, l'homoarginine (comme composant de la caséine après guanidylation) et le xylose, également ajoutés au lait. II est apparu que l'absorption de la $3 \mathrm{MH}$ in vitro dépend de la dose et a été inhibée par la leucine, ce qui indique qu'elle est transportée comme les acides aminés neutres. Après l'ingestion de lait apportant des quantités de $3 \mathrm{MH}$ de 2,5 à $20 \mu \mathrm{mol} / \mathrm{kg}$ de poids vif, la concentration plasmatique de $3 \mathrm{MH}$ a augmenté en fonction de la dose; la valeur maximale a été atteinte entre 3 et $8 \mathrm{~h}$. La concentration de lysine a suivi initialement le cours observé pour la $3 \mathrm{MH}$, mais a diminué plus rapidement. La concentration d'homoarginine a commencé à monter seulement de 2 à $4 \mathrm{~h}$ après l'ingestion du lait, probablement à cause du temps nécessaire à la dégradation de la caséine. La concentration de xylose a augmenté dans le sang aussi vite que celle de $3 \mathrm{MH}$ et de la lysine. La distribution d'un aliment contenant des protéines de soja durant 36 j et l'administration de néomycine aux veaux nouveau-nés pendant $5 \mathrm{j}$ ont induit une malabsorption de xylose. En revanche, l'absorption de la $3 \mathrm{MH}$ et la digestion de la caséine, appréciée d'après l'absorption de l'homoarginine, n'ont pas été réduites.
\end{abstract}

veau / (mal)absorption / 3-méthylhistidine / homoarginine / lysine / xylose

Summary - Post-prandial changes in blood plasma of 3-methylhistidine related to lysine, homoarginine and xylose under normal conditions and during malabsorption in pre-ruminant calves. We have studied the absorption of 3-methylhistidine (3MH) in vitro and changes in its plasma concentration in preruminant calves after ingestion of milk containing 3MH under normal and malabsorptive conditions. Changes in $3 \mathrm{MH}$ concentration were compared to those in lysine, homoarginine (as a component of guanidinated caseine) and xylose, also added to milk. The absorption of $3 \mathrm{MH}$ in vitro was dose-dependent and was inhibited by leucine, indicating that $3 \mathrm{MH}$ was transported in the same manner as neutral amino acids. After the ingestion of milk supplying 2.5-20 $\mu \mathrm{mol}$

\footnotetext{
* Correspondance et tirés à part
} 
(3MH)/kg body weight, $3 \mathrm{MH}$ increased dose-dependently within $1.75 \mathrm{~h}$ and reached maximal levels between 3 and $8 \mathrm{~h}$. Lysine concentration initially parallelled the course of blood $3 \mathrm{MH}$ levels but dropped sooner. Homoarginine concentration did not increase until 2-4 h postprandially, probably as a consequence of the time needed for casein degradation. Xylose concentration increased as rapidly as $3 \mathrm{MH}$ and lysine concentration. Feeding a diet containing soybean protein for $36 \mathrm{~d}$ and administration of neomycine for $5 d$ to newborn calves induced malabsorption of xylose. However, 3MH absorption and caseine digestion, which increased after homoarginine absorption were not reduced.

calf / (mal)absorption / 3-methylhistidine / homoarginine / lysine / xylose

\section{INTRODUCTION}

Chez le veau pré-ruminant, les diarrhées sont souvent combinées avec une malabsorption, à la suite d'infections causées par exemple par les corona- et rotavirus (Woode et al, 1978) ou par des aliments particuliers. Les études précédentes dans des situations de malabsorption ont porté sur l'absorption des glucides (Celeda et al, 1983). Pour tester, dans des conditions cliniques, la digestion des protéines et l'absorption des acides aminés, on peut utiliser des protéines, peptides et acides aminés et étudier l'évolution des taux d'acides aminés libres dans le sang pendant l'absorption. La 3-méthylhistidine (3MH) présente les caractéristiques nécessaires pour être utilisée comme indicateur de l'absorption intestinale. Une fois absorbée ou libérée des tissus et circulant dans le sang, la 3MH n'est plus métabolisée et est éliminée quantitativement par les reins chez les bovins, comme chez l'homme (Asatoor et Armstrong, 1967; Young et al, 1972; Harris et Milne, 1981; Bachmann et al, 1984). En outre, la $3 \mathrm{MH}$ n'est guère présente dans l'alimentation des bovins (Gur-Chiang et Bergen, 1986). Nous avons donc comparé l'évolution des concentrations postprandiales de $3 \mathrm{MH}$ à celle des concentrations de lysine libre (Lys), l'homoarginine (HA) apportée par de la caséine guanidylée (Maga, 1981; Hagemeister et Ebersdobler, 1985) et de xylose
$(X y l)$, en conditions physiologiques. En outre, nous avons vérifié l'aptitude de la $3 \mathrm{MH}$, de I'HA et du $X y \mid$ à mettre en évidence des altérations de l'intestin grêle, en utilisant de la néomycine (Rollin et al, 1986) et un lait de remplacement contenant des protéines de soja (Sissons et Smith, 1976; Seegraber et Morrill, 1979; Pedersen et Sissons, 1984; Grant et al, 1989) pour provoquer une malabsorption chez des veaux pré-ruminants.

\section{MATÉRIEL ET MÉTHODES}

\section{Absorption de $3 M H$ in vitro}

Nous avons déterminé l'absorption de la $3 \mathrm{MH}$ en utilisant la méthode des sacs intestinaux inversés (Wilson et Wiseman, 1954). Les sacs ont été formés à partir de jéjunum moyen de rats. Ces rats ont reçu un régime sans viande. Ils ont été euthanasiés à l'éther. Chaque expérience a été basée sur 5 répétitions. Nous avons réalisé différents types d'essais pour déterminer par quel type de transporteurs la $3 \mathrm{MH}$ est absorbée. Selon l'essai, les sacs contenant respectivement :

- tampon Krebs;

$-100 \mu \mathrm{mol} / \mathrm{l}$ de $3 \mathrm{MH}$ dans du tampon Krebs;

$-500 \mu \mathrm{mol} / \mathrm{l}$ de $3 \mathrm{MH}$ dans du tampon Krebs;

$-100 \mu \mathrm{mol} / \mathrm{l}$ de $3 \mathrm{MH}+5 \mathrm{mmol} / \mathrm{l}$ de L-leucine dans du tampon Krebs;

- essais de contrôle avec $100 \mu \mathrm{mol} / / \mathrm{lde}\left({ }^{3} \mathrm{H}\right)$ L-leucine $(3 \mu \mathrm{Ci} / 100 \mathrm{ml})$. 
Ce test a permis d'évaluer la qualité des sacs intestinaux.

\section{Tests d'absorption in vivo : veaux nourris au lait}

Douze veaux pré-ruminants (Simmental $\times$ Holstein Rouge ou Brune Suisse de poids vif entre 50 et $95 \mathrm{~kg}$ ) ont été nourris exclusivement au lait, de manière individuelle, 2 fois par $j$, avant les tests d'absorption. La veille de l'essai, un cathéter a été placé dans une veine jugulaire externe. Le sang a été prélevé dans des tubes contenant de l'héparinate de lithium (Milian SA, $\mathrm{CH}-1228$ Plan-les-Ouates) et centrifugé $15 \mathrm{~min}$ à $1500 \mathrm{~g}$. Le premier jour du test d'absorption, les veaux auxquels on a donné l'HA ont reçu du lait apportant $0,15 \mathrm{~g}$ de caséine par $\mathrm{kg}$ de poids vif (PV). Le lendemain, l'expérience a été répétée en ajoutant 2, 5, 10 ou $20 \mu \mathrm{mol} / \mathrm{kg}$ (PV) de $3 \mathrm{MH}, 0,5 \mathrm{~g}$ de $\mathrm{Xyl}$ et $70 \mu \mathrm{mol} / \mathrm{kg}$ (PV) de HA ou $0,3 \mathrm{~g} / \mathrm{kg}(\mathrm{PV})$ de Lys.

\section{Tests d'absorption in vivo : influence de l'administration de néomycine}

Seize veaux nouveau-nés (Simmental $x$ Holstein Rouge ou Brune Suisse) ont été nourris au colostrum maternel pendant les 2 premiers jours, puis avec 1,5 I de lait 2 fois par jour. $\AA$ partir de l'âge de 3-6 j, 8 d'entre eux ont reçu une solution aqueuse de sulfate de néomycine (14\%; $0,25 \mathrm{ml} / \mathrm{kg} \mathrm{PV}$ ) par voie orale 3 fois par j (à $7 \mathrm{~h}, 15 \mathrm{~h}$ et $23 \mathrm{~h}$ ) pendant $5 \mathrm{j}$. Le sixième jour, $10 \mu \mathrm{mol} / \mathrm{kg}$ (PV) de $3 \mathrm{MH}, 0,5 \mathrm{~g} / \mathrm{kg}$ (PV) Xyl et $90 \mu \mathrm{mol} / \mathrm{kg}$ (PV) de HA ont été ajoutés au lait pour tester leur absorption. Le sang a été prélevé dans des vacutainers héparinisés (Sherwood Medical, St Louis, MO, USA).

\section{Tests d'absorption in vivo : influence des protéines de soja}

Douze veaux mâles (Simmental $x$ Holstein Rouge ou Holstein Pie Noire de 50 à $80 \mathrm{~kg}$ PV) ont été répartis selon leur poids en 2 groupes de 6. Trois aliments d'allaitement ont été utilisés. Ils contenaient 0,7 ou $15,5 \%$ de protéines de soja. Les teneurs en matière sèche (MS; $94,9 \%$ ) et (sur base de la MS) en matière azotée $(22,5 \%)$, en cellulose brute $(0,5 \%)$, en graisse brute

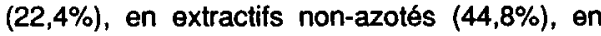
cendres brutes $(7,7 \%)$ et en énergie $(11,3 \mathrm{MJ})$ des 3 aliments étaient pratiquement égales. Les veaux ont été nourris au seau individuellement 2 fois par jour. La première semaine, ils ont été habitués à l'aliment témoin dépourvu de protéines de soja. Pour induire une malabsorption, l'aliment le plus riche en protéines de soja (15\%) a été administré les lundi de la deuxième, quatrième et sixième semaine et l'aliment contenant $7 \%$ de protéines de soja, les lundi de la troisième et cinquième semaine et les mercredi et vendredi de la deuxième à la cinquième semaine. Entre temps, les veaux ont reçu l'aliment témoin. Le groupe de contrôle a été nourri en permanence avec l'aliment témoin. Le jour suivant l'administration de l'aliment le plus riche en protéines de soja en sixième semaine, les 12 veaux ont reçu par $\mathrm{kg}(\mathrm{PV}) 10 \mu \mathrm{mol}$ de $3 \mathrm{MH}, 55$ $\mu \mathrm{mol}$ de $\mathrm{HA}$ et $0,5 \mathrm{~g}$ de $X y l$ ajoutés dans l'aliment témoin. Le sang a été prélevé dans des vacutainers.

\section{Aliments}

Les veaux ont été nourris avec du lait de vache, sauf dans l'essai effectué avec le soja. La farine de soja (produit $n^{\circ} 91-11$; Kliba, Moulin Klingental SA, CH-4303 Kaiseraugst) a subi un traitement hydrothermique (température atteinte dans le produit : $19{ }^{\circ} \mathrm{C}$ pendant $8 \mathrm{~min}$ ) qui a réduit l'activité de l'uréase (en-dessous de $0,02 \mathrm{mg}$ d'azote $\cdot \mathrm{g}^{-1} \cdot \mathrm{min}^{-1}$ à $30^{\circ} \mathrm{C}$ ), de la lipase, de la péroxydase et celui des inhibiteurs de la trypsine. Les teneurs moyennes en matière azotée, cellulose brute, matière grasse, extractif non azoté et cendres étaient respectivement de $40,7,3,2,23,3,27,2$, et $5,6 \%$. Les teneurs moyennes en globuline, albumine, protéose et azote non protéique (de l'azote total) étaient respectivement de $84,5,4,4,4$ et $6 \%$.

\section{Produits chimiques pour les tests d'absorption}

La L-leucine et la L-3MH provenaient de chez Sigma Chemical Company, St Louis, la $\left({ }^{3} \mathrm{H}\right) \mathrm{L}-$ leucine de chez New England Nuclear Chemi- 
cals, CH-6072 Dreieich, la Lys de chez Selectchemie AG, CH-8038 Zürich, le Xyl de chez Fluka Chemie AG, CH-9470 Buchs, et la néomycine de chez Siegfried AG, CH-4800 Zofingen. La caséine contenant de l'HA (Lys transformée en HA par guanidylation; Maga 1981) a été préparée à la Bundesanstalt für Milchforschung, D-2300 Kiel.

\section{Analyses}

\section{Dosage de la 3MH}

Elle a été dosée par radio-immunologie (Bachmann et al, 1984; Blum et al, 1985). Les concentrations de Lys et de HA ont été déterminées par chromatographie sur résine échangeuse d'ions, après déprotéinisation du plasma à l'aide d'un volume égal de solution contenant $200 \mathrm{~g} /$ d'acide sulfosalicylique.

\section{Dosage de l'azote $\alpha$-aminié, des protéines, de l'albumine du xylose et du glucose}

IIs ont été dosés respectivement selon les techniques de Goodwin (1968), Richterich et Colombo (1978), Doumas et al (1971) et Richterich et Colombo (1978) pour les 4 premières substances; la teneur en glucose a été déterminée à l'aide d'un kit Galenopharm ( $\mathrm{CH}-2300$ La Chaux-de-Fonds).

\section{Détermination des anticorps plasmatiques dirigés contre les protéines de soja ingérées}

Ils ont été déterminés d'après Hawkes et al (1981). Comme antigène, on a utilisé les protéines de soja dans l'aliment d'allaitement. Un microlitre d'une solution de soja par dot (2,5-10 $\mathrm{mg}$ de protéine par $\mathrm{ml}$ ) a été transféré sur des filtres de nitrocellulose (Type HA, 0,45 $\mu \mathrm{m}$, cat HAWG 304 FO, Millipore Corp, Bedford, CA, USA). Pour éviter une contamination avec des protéines autres que celles du soja, les filtres ont été bloqués par une solution de caséine (1,5 $\mathrm{mg} / \mathrm{ml}$ de tampon salin de Tris; TBS). Après $1 \mathrm{~h}$ d'incubation, la nitrocellulose a été coupée en bandes et séchée à température ambiante. Ensuite, ces bandes ont été incubées sous une faible agitation dans des réservoirs, avec différentes dilutions de sérum (1:200-1:2000). Le lendemain, les réservoirs ont été rincés 3 fois avec du TBS. Ensuite, $1 \mathrm{ml}$ d'anticorps de lapinanti-IgG de bovin dilué, conjugué avec de la péroxidase (Bio Yeda, Israël), a été ajouté et incubé pendant $4 \mathrm{~h}$. L'opération de lavage au TBS a été répétée avant l'ajout de $1 \mathrm{ml}$ d'une solution de chromogène [1 part 4-chloro-1-naphtol à $0,3 \%$ dans du méthanol, 5 parts de TBS et de 5-10 $\mu \mathrm{l}$ de $\mathrm{H}_{2} \mathrm{O}_{2} 30 \%$ ] pour visualiser la réaction enzymatique. Après un dernier lavage avec de l'eau distillée et le séchage, l'intensité des dots bleus a été mesurée avec un scanner densitométrique (Camag TLC-Scanner, $\mathrm{CH}-4132$ Muttenz). Les résultats sont donnés en densité optique relative (ROD).

\section{Analyses statistiques}

In vitro, les résultats ont été comparés par le test de Mann-Whitney.

In vivo, les concentrations postprandiales ont été comparées avec la valeur moyenne basale. Les réactions totales pendant 9 ou $24 \mathrm{~h}$ des différents essais $(\Delta 0-9 h$ ou $\Delta 0-24 h)$ ont été évaluées d'après les surfaces situées sous les courbes de concentration et comparées par le test de Kruskal-Wallis. Les résultats ont été exprimés sous la forme de moyennes \pm écart type) des échantillons.

\section{RÉSULTATS}

\section{Absorption de $3 M H$ in vitro (tableau I)}

L'absorption muco-séreuse a augmenté avec la concentration initiale de $3 \mathrm{MH}$. Quand on a ajouté $5 \mathrm{mmol}$ de leucine à la solution contenant $118 \mu \mathrm{mol} / \mathrm{l}$ de $3 \mathrm{MH}$, l'absorption de $3 \mathrm{MH}$ a été beaucoup plus faible. L'absorption de leucine en l'absence de $3 \mathrm{MH}$ a été importante. Toutes les concentrations séreuses finales ont été 
Tableau I. Absorption de 3MH in vitro.

\begin{tabular}{|c|c|c|c|c|c|}
\hline \multicolumn{3}{|c|}{ Concentration initiales } & \multicolumn{2}{|c|}{ Concentrations $3 \mathrm{MH}$ finales } & \multirow{2}{*}{$\begin{array}{c}\text { Absorption } 1 \\
(\mu \mathrm{mol} / 100 \mathrm{mg} \mathrm{MS} \\
145 \mathrm{~min})\end{array}$} \\
\hline & $\begin{array}{c}3 \mathrm{MH} \\
(\mu \mathrm{mol} / \mathrm{l})\end{array}$ & $\begin{array}{l}\text { Leucine } \\
\text { ( } \mu \text { moll/I) }\end{array}$ & $\begin{array}{c}\text { muqueux } \\
(\mu \text { mol/l) }\end{array}$ & $\begin{array}{l}\text { séreux } \\
(\mu \text { mollI) }\end{array}$ & \\
\hline A & 6,5 & 0 & $0,13 \pm 0,06^{a}$ & $5,4 \pm 2,3^{b}$ & $12,0 \pm 2,7$ \\
\hline B & 100 & 0 & $88 \pm 3^{a}$ & $301 \pm 45^{b}$ & $232 \pm 34$ \\
\hline C & 498 & 0 & $453 \pm 16^{a}$ & $927 \pm 105^{b}$ & $463 \pm 47$ \\
\hline D & 118 & 5000 & $113 \pm 3^{a}$ & $121 \pm 3^{a}$ & $16 \pm 3$ \\
\hline E & 0 & 5000 & $56 \pm 5^{a}$ & $665 \pm 30^{b}$ & $698 \pm 63$ \\
\hline
\end{tabular}

1 Exp A-D: $3 M H ;$ Exp E: $\left({ }^{3} \mathrm{H}\right)$-Leucine; MS, matière sèche; ${ }^{a, b}$ Les valeurs accompagnées de leftres différentes sont significativement différentes $(P<0,05)$.

plus élevées que les concentrations muqueuses $(P<0,05)$, à l'exception de celle de $3 \mathrm{MH}$ incubée en présence d'une forte concentration de leucine.

\section{Tests l'absorption in vivo : veaux nourris au lait (fig 1)}

Les valeurs basales des paramètres de traitements n'ont pas été significativement différentes les unes des autres. Après le repas, la concentration plasmatique de $3 \mathrm{MH}$ a augmenté immédiatement et linéairement avec la quantité ajoutée au lait $(r=0,93 ; P<0,01)$ ou avec la dose par $\mathrm{kg}$ (PV) $(r=0,95 ; P<0,01)$; le maximum a été atteint entre 3 et $6 \mathrm{~h}$. Avec le lait non additionné de $3 \mathrm{MH}$, la concentration a diminué légèrement. La concentration de Lys a peu varié dans le groupe recevant le lait seul mais a immédiatement augmenté chez les animaux qui ont ingéré un supplément de Lys $(P<0,05)$. Le taux d'HA n'a augmenté qu'à partir de $2 \mathrm{~h}$ après l'ingestion et le maximum a été atteint à $9 \mathrm{~h}$. Le taux d'HA d'un veau traité avec une dose 10 fois plus élevée que les autres n'a monté que 1,5 h après l'ingestion (non présenté). L'HA n'a pu être détectée dans le plasma des veaux témoins. La concentration postprandiale de l'azote $\alpha$-aminé (mesurée seulement chez les témoins; non présentée) a eu tendance à monter, tandis que les taux de protéines et d'albumine (non présentés) ont baissé, mais non significativement. Dans le groupe témoin, le taux de $X y l$ est resté très stable. La concentration de $X y l$ a augmenté immédiatement après l'administration et est redescendue après 4-5 h. Le taux de glucose a augmenté immédiatement après le repas, mais est revenu à la valeur basale après 4-6 h. II a à nouveau augmenté après le repas du soir.

Tests d'absorption in vivo : influence de l'administration de néomycine (fig 2)

Aucune différence pour les valeurs basales n'a été observée entre les 2 groupes 

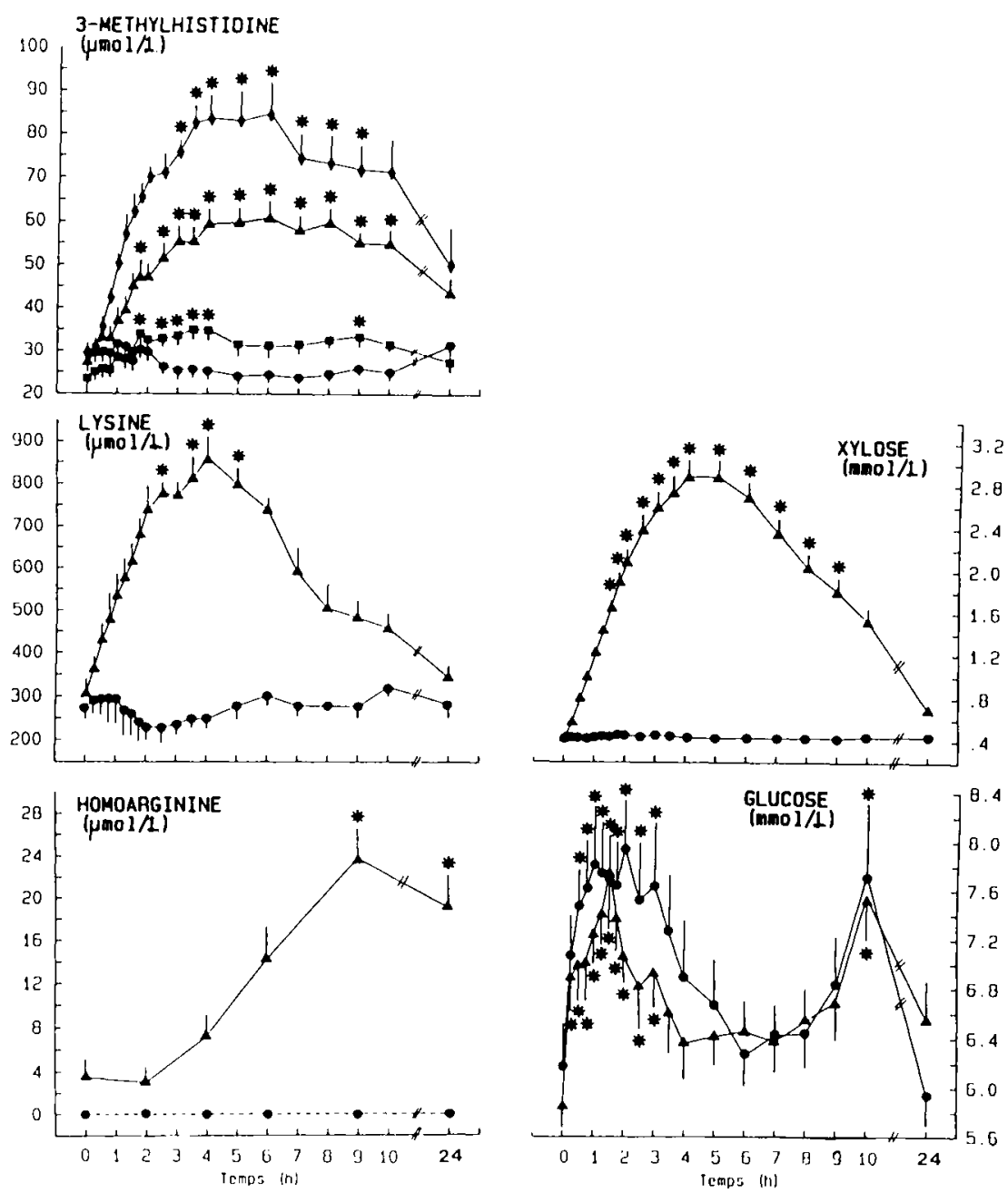

Fig 1. Tests d'absorption chez des veaux pré-ruminants recevant du lait entier : $\longrightarrow$ - témoin, lait seul; -1 lait $+2,5 \mu \mathrm{mol}$ de $3 \mathrm{MH} / \mathrm{kg}$ de poids vif (PV); $-\mathbf{A}-$ lait $+10 \mu \mathrm{mol}$ de $3 \mathrm{MH}+0,5 \mathrm{~g}$ de $\mathrm{D}+\mathrm{xylose}+70 \mu \mathrm{mol} \mathrm{HA}$ ou $0,3 \mathrm{~g}$ de lysine/kg (PV); - — lait $+20 \mu \mathrm{mol}(3 \mathrm{MH}) \mathrm{kg}$ PV; -.-.$--\mathrm{HA}$ audessous de la sensibilité de la détection. ": significativement différent de la valeur basale $(P<0,05)$.

sauf dans le cas du glucose, dont le taux a été plus élevé chez le groupe témoin $(P<0,05)$. Le taux de $3 \mathrm{MH}$ est monté immédiatement après le repas et a atteint un maximum entre 6 et $9 \mathrm{~h}$. Celui de Lys n'a pas significativement varié. Celui d'HA a augmenté à partir de $2 \mathrm{~h}$. Les concentra- tions de protéines et d'albumine ont eu tendance à baisser après le repas et sont revenues au niveau basal après $9 \mathrm{~h}$ (non présenté). Aucune des surfaces situées sous les courbes de 0 à $9 \mathrm{~h}(\Delta 0-9 \mathrm{~h})$ n'a été significativement influencée par le traitement, en ce qui concerne le $3 \mathrm{MH}$, la Lys, 


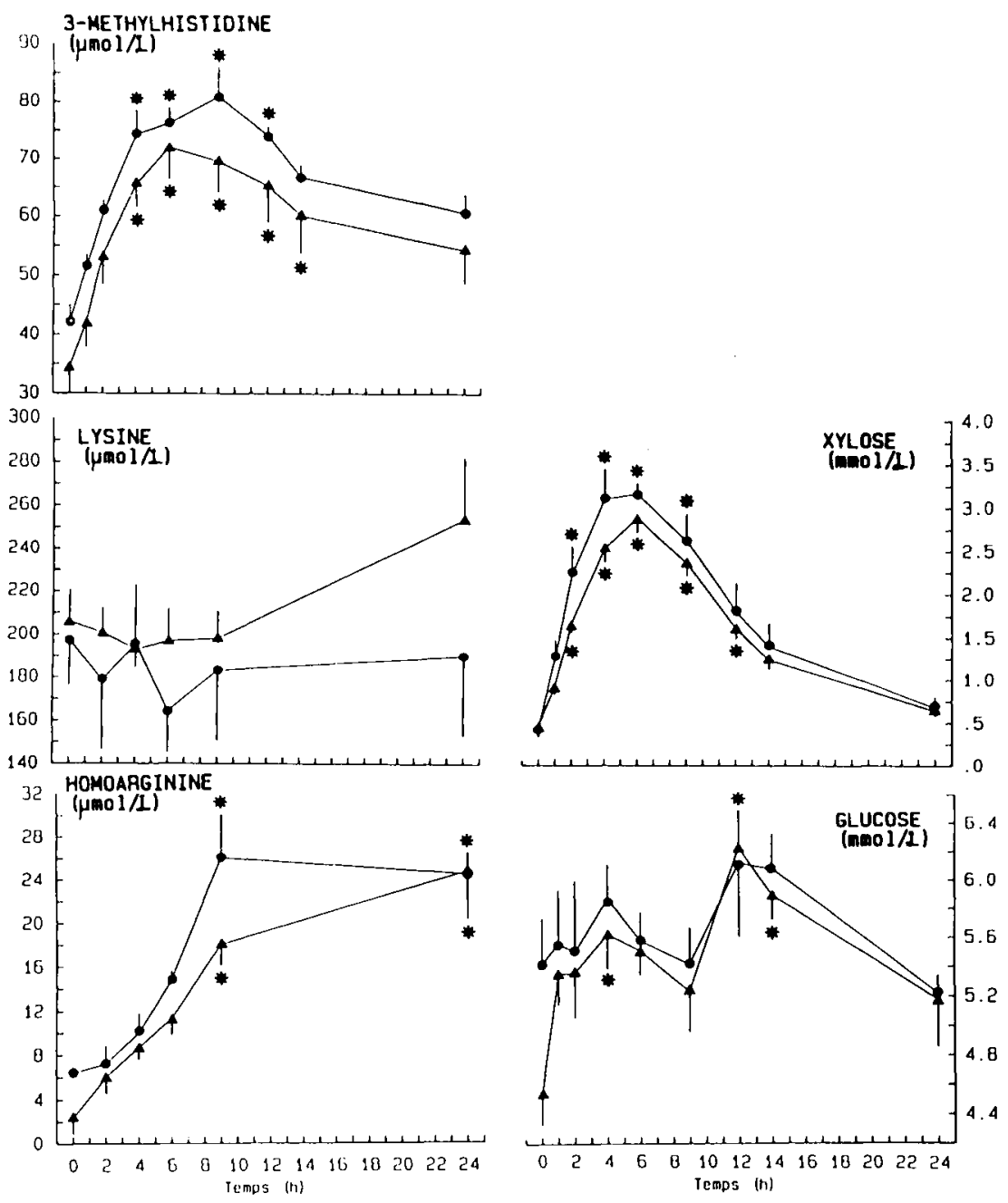

Fig 2. Tests d'absorption chez des veaux nouveau-nés après administration de néomycine : groupe témoin; - $\Delta-$ groupe traité à la néomycine. Les veaux des 2 groupes ont reçu $1,5 \mathrm{~kg}$ de lait $+10 \mu \mathrm{mol}(3 \mathrm{MH}) \mathrm{kg}(\mathrm{PC})+90 \mu \mathrm{mol}(\mathrm{HA}) \mathrm{kg} \mathrm{PC}+0,5 \mathrm{~g}$ de D+xylose $/ \mathrm{kg}$ (PV). Les valeurs de xylose jusqu'à $9 \mathrm{~h}$ après le repas ont été significativement différentes entre le groupe témoin et le groupe traité à la néomycine $(P<0,05)$. * Significativement différent de la valeur basale $(P<0,05)$.

l'HA, les protéines et l'albumine. Le taux de $X y l$ a augmenté immédiatement après le repas et a diminué après $6 \mathrm{~h}$. Toutes les valeurs observées jusqu'à $9 \mathrm{~h}$ après le repas ont été inférieures dans le groupe recevant de la néomycine à celles mesurées dans le groupe témoin $(P<0,05)$; il en a été de même pour la $\Delta 0-9 \mathrm{~h}$. Le taux de glucose a augmenté plus fortement chez le groupe traité à la néomycine où il a été significativement différent de la valeur basale après $4 \mathrm{~h}(P<0,05)$. La $\Delta 0-24 \mathrm{~h}$ a été plus grande chez le groupe traité à la néomycine $(P<0,05)$. 


\section{Tests d'absorption in vivo : influence des protéines de soja (fig 3)}

Aucune différence n'a été observée entre les 2 groupes pour les valeurs basales, sauf celles de la Lys et des protéines (non présenté), qui ont été plus basses dans le groupe ayant reçu des protéines de soja $(P<0,05)$. Après le repas, la concentration de $3 \mathrm{MH}$ a augmenté immédiatement dans les 2 groupes. La $\Delta 0-9$ h et la $\Delta 0-$ $24 \mathrm{~h}$ ont été plus marquées dans le groupe soja, mais les différences n'ont pas été significatives. Dans le groupe témoin, le taux de Lys est resté constant, tandis que dans le groupe soja, il a augmenté jusqu'à $6 \mathrm{~h}$ après le repas et est devenu différent du taux basal après $24 \mathrm{~h}(P<$ $0,05)$. La $\Delta 0-9 \mathrm{~h}$ a été plus élevée dans le groupe soja $(P<0,05)$. Le taux d'HA a augmenté plus rapidement après $4 \mathrm{~h}$ dans le groupe soja que dans le groupe témoin. La $\Delta 0-24 \mathrm{~h}$, mais pas la $\Delta 0-9 \mathrm{~h}$, a été plus élevée dans le groupe soja $(P<$ $0,05)$. Les taux de protéines et d'albumine ont diminué dans les 2 groupes, mais non significativement (non présenté). La concentration de $X y l$ a augmenté plus nettement dans le groupe témoin que dans le groupe soja. La $\Delta 0-9 \mathrm{~h}$ a été plus grande dans le groupe témoin $(P<0,05)$. Le taux de glucose a augmenté initialement plus fortement dans le groupe soja, mais la $\Delta$ 0-9 h n'a pas été différente entre les 2 groupes. Au début du test d'absorption et $24 \mathrm{~h}$ plus tard, les titres anticorps plasmatiques anti-soja ont été considérablement plus élevés dans le groupe soja (69 \pm 6 et $76 \pm 4$ ROD, respectivement) que dans le groupe témoin ( $12 \pm 3$ et $9 \pm 2$ ROD, respectivement) avec des dilutions de sérum au $1 / 300(P<0,01)$.

\section{DISCUSSION}

À notre connaissance, l'absorption de $3 \mathrm{MH}$ in vitro n'a pas été étudiée auparavant. Nos expériences montrent que la quantité de $3 \mathrm{MH}$ absorbée dépend de la concentration initiale. En outre, la $3 \mathrm{MH}$ est absorbée par le système des acides aminés neutres puisque le leucine a inhibé le passage de la $3 \mathrm{MH}$ (Scharrer, 1978). Nos conditions expérimentales semblent avoir été satisfaisantes d'après les résultats obtenus avec la leucine. In vivo, les variations des concentrations plasmatiques postprandiales des différentes substances étudiées ont été liées à la vitesse de digestion et d'absorption, mais ont également été influencées par le métabolisme intermédiaire et par l'excrétion urinaire et endogène.

Les veaux traités à la néomycine n'ont pas présenté de fortes diarrhées. Au contraire, une diarrhée marquée a été visible chez 5 des 6 veaux recevant le régime contenant du tourteau de soja. Cela était probablement dû à l'effet nocif de ces protéines sur la muqueuse intestinale ( $\mathrm{Pe}$ dersen et Sissons, 1984). Les titres élevés d'anticorps dirigés contre les protéines de soja chez les veaux recevant le régime soja indiquent qu'il s'est produit une réaction immunitaire, comme l'ont observé Kilshaw et Sissons (1979) par hémagglutination passive. Sur la base des résultats obtenus avec le Xyl, l'administration de néomycine et de soja a induit une malabsorption, comme l'ont observé Sissons et Smith (1976), Seegraber et Morrill (1979), Pedersen et Sissons (1984), Rollin et al (1986) et Grant et al (1989).

La concentration de $3 \mathrm{MH}$ dans le lait de vache est faible car le taux de $3 \mathrm{MH}$ a dimi- 

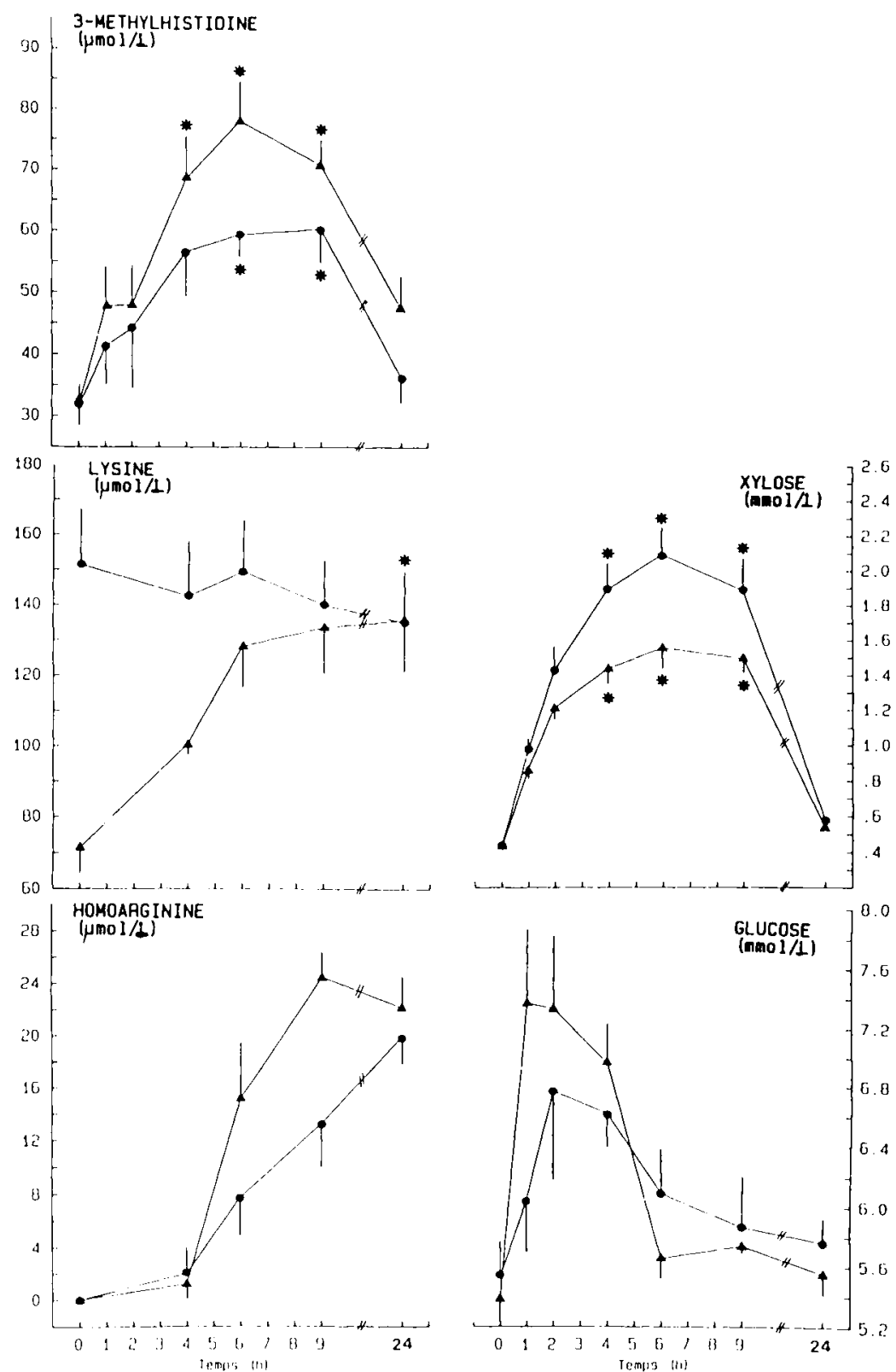

Fig 3. Tests d'absorption chez des veaux pré-ruminants nourris avec un aliment contenant du soja : - groupe témoin; - $-\mathbf{A}$ - groupe traité au soja. Les veaux des 2 groupes ont reçu $10 \mu \mathrm{mol}$ $(3 \mathrm{MH}) \mathrm{kg}+55 \mu \mathrm{mol}(\mathrm{HA}) \mathrm{kg}+0,5 \mathrm{~g}$ (D+xylose) par $\mathrm{kg}$ poids vif (PV). Les valeurs de xylose jusqu'à $9 \mathrm{~h}$ après le repas ont été significativement différentes entre le groupe témoin et le groupe traité au soja $(P<0,05) .{ }^{*}:$ significativement différent lie la valeur basale $(P<0,05)$. 
nué après l'ingestion de lait sans adjonction de 3MH. Bachmann et al (1984) ont aussi constaté chez l'homme une bonne corrélation entre la dose administrée et la concentration postprandiale dans le sang. Chez le veau, la plus forte concentration de $3 \mathrm{MH}$ a été atteinte entre $3 \mathrm{~h}$ et $8 \mathrm{~h}$, tandis que chez l'homme, le maximum se situait après $1,25 \mathrm{~h}$. Cette différence a pu résulter de la libération progressive de la $3 \mathrm{MH}$ après coagulation du lait dans la caillette. Les concentrations postprandiales de $3 \mathrm{MH}$ dans le groupe traité à la néomycine ou au soja ont été comparables à celles du groupe témoin. La $3 \mathrm{MH}$ ne semble donc pas pouvoir être utilisée comme indicateur sensible d'une altération de la paroi de l'intestin grêle.

Dans des conditions normales, le taux de Lys est resté relativement constant après l'ingestion de lait sans supplément. L'augmentation rapide observée après l'ingestion de lait additionné de Lys est en accord avec les résultats de Tzeng et Davis (1980). Elle indique que l'évacuation gastrique de la Lys libre est probablement beaucoup plus rapide que celle de la Lys incluse dans les protéines. La Lys est métabolisée dans les tissus, ce qui pourrait expliquer son retour à la valeur basale plus rapide que celui de $3 \mathrm{MH}$. Comme celle de $3 \mathrm{MH}$, la concentration postprandiale de Lys n'a été influencée ni par la néomycine ni par l'introduction de soja dans l'aliment. La veille du test, l'administration du régime soja a provoqué une forte diarrhée, diminuant probablement l'absorption de Lys et expliquant la valeur basale relativement basse le jour du test d'absorption chez ces veaux.

Après l'ingestion de lait supplémenté en caséine guanidylée, la rétention dans la caillette et le temps nécessaire pour digérer la caséine (Ternouth et al, 1974; Yvon et al, 1986) pourraient expliquer l'augmentation tardive du taux plasmatique de I'HA par rapport à la $3 \mathrm{MH}$ et la Lys. Puisque les variations postprandiales du taux de HA n'ont pas été affectées par la néomycine, il est difficile d'expliquer le fait que le taux d'HA ait été accru chez les veaux recevant le régime soja.

Notre étude a démontré que le $X y l$ est normalement présent en concentration très faible et peu variable dans le plasma des veaux. Distribué dans le lait, à raison de $0,5 \mathrm{~g}$ par $\mathrm{kg}(\mathrm{PV})$, son taux a augmenté immédiatement après le repas, en parallèle avec le taux de $3 \mathrm{MH}$ et de Lys, suggérant que les 3 substances ont des vitesses d'évacuation gastrique voisines. Avec la même dose de $X y l$, distribué en solution aqueuse à $10 \%$, Seegraber et Morrill (1979) ont observé un maximum plus élevé et plus précoce que le nôtre. La compétition pour l'occupation des transporteurs intestinaux entre le $\mathrm{Xyl}$ et le lactose dans le lait pourrait expliquer ces différences (Csaky et Ho, 1965). Après avoir atteint son maximum, la concentration de $X y l$ a baissé plus rapidement que celle de $3 \mathrm{MH}$, probablement à cause d'une métabolisation et d'une excrétion différente. Après le repas, les taux plasmatiques de $X y l$ du groupe traité à la néomycine ont été moins élevés que ceux du groupe témoin. La néomycine a donc provoqué une malabsorption, en accord avec Rollin et al (1986). Il en a été de même chez les veaux du groupe soja, confirmant ainsi la malabsorption observée notamment par Segraber et Morrill (1979) avec ce type de régime.

La concentration du glucose a augmenté après le repas, en accord avec Chapman et al (1986) et Blum et Flückiger (1988). Elle est revenue plus rapidement à la valeur basale que celle de $3 \mathrm{MH}$, Lys, HA et Xyl. Cette chute rapide pourrait s'expliquer par la sécrétion postprandiale d'insuline (non présenté) qui stimule le transport du glucose dans les tissus. Le taux 
basal de glucose du groupe recevant de la néomycine a été diminué de manière encore plus radicale que dans les essais de Rollin et al (1986). Le glucose a paru être mieux absorbé dans le groupe soja que dans le groupe témoin et a suivi la même tendance que la $3 \mathrm{MH}$, la Lys et l'HA, mais s'est comporté différemment du Xyl.

En conclusion, les essais in vitro nous ont permis de constater que la $3 \mathrm{MH}$ a suivi le même mode d'absorption que les acides aminés neutres. Contrairement au $\mathrm{XYl}$, la $3 \mathrm{MH}$ et la Lys ne semblent pas pouvoir être utilisées comme indicateurs suffisamment sensibles de la malabsorption causée par la néomycine et les protéines de soja chez des veaux pré-ruminants. Le comportement de l'HA dans le plasma sanguin a été semblable à celui de la $3 \mathrm{MH}$, mais la dégradation assez lente de la caséine guanidylée a été responsable pour un délai considérable de l'absorption de I'HA relative au $3 \mathrm{MH}$.

\section{REMERCIEMENTS}

Les auteurs remercient l'Office vétérinaire fédéral pour le financement du projet ainsi que le Prof H Hagemeister de l'Inst für Physiologie und Biochemie der Ernährung, Bundesanstalt für Milchforschung, D-2300 Kiel, le Prof E Scharrer et le $\mathrm{Dr} S$ Wolffram de l'Inst für Veterinärphysiologie der Universität, $\mathrm{CH}-8057$ Zürich et le PD Dr B Stadler de l'Inst für Klinische Immunologie der Universität, $\mathrm{CH}-3000$ Bern pour leur collaboration.

\section{RÉFÉRENCES}

Asatoor AM, Armstrong MD (1967) 3methylhistidine, a component of actin. Biochem Biophys Res Commun 26, 168-174

Bachmann K, Galeazzi R, Millet A, Burger AG (1984) Plasma levels of 3-methylhistidine after ingestion of the pure amino acid or muscular protein measured by radioimmunoassay. Metabolism 33, 107-110
Blum JW, Flückiger N (1988) Early metabolic and endocrine effects of perorally administered $\beta$-adrenergic agonists in calves. Eur $J$ Pharmacol 151, 177-187

Blum JW, Reding T, Jans F, Wanner M, Zemp $M$, Bachmann $K$ (1985) Variations of 3methylhistidine in blood of dairy cows. $J$ Dairy Sci 68, 2580-2587

Celeda L, Cerny J, Fendrich Z, Senius K, Rouchal J, Janku I, Kvetina J, Raskova H, Kubicek $A$, Polak $L$ (1983) Xylose absorption in normal and diarrhoeic calves. Zentralb/ Veterinaermed B30, 189-194

Chapman HW, Butler RDG, Newell M (1986) The route of liquids administered to calves by esophageal feeder. Can J Vet Res 50, 84-87

Csaky TZ, Ho PM (1965) Intestinal transport of D+Xylose. Proc Soc Exp Biol Med 120, 403408

Doumas BT, Watson WA, Biggs HG (1971) Albumin standards and the measurement of serum albumin with bromcresol green. Clin Chim Acta 31, 87-96

Goodwin JF (1968) On the measurement of urinary amino nitrogen with 1-fluoro2,4, dinitrobenzene. Clin Chim Acta 21, 231235

Grant AL, Holland RE, Thomas W, King KJ, Wiesman JS (1989) Effects of dietary amines on the small intestine in calves fed soybean protein. J Nutr 119, 1.034-1041

Gur-Chiang PWT, Bergen WG (1986) Nmethylhistidine - Feed and duodenal content and ruminal degradation. $J$ Anim Sci 62, 1713-1718

Hagemeister $H$, Ebersdobler $H$ (1985) Chemical labelling of dietary protein by transformation of lysine to homoarginine - a new technique to follow intestinal digestion and absorption. Proc Nutr Soc 44, 133A

Harris $\mathrm{Cl}$, Milne $\mathrm{G}$ (1981) The urinary excretion of 3-methylhistidine by cattle: validation as a index of muscle protein breakdown. Br J Nutr $45,411-421$

Hawkes R, Niday E, Gordon J (1981) A dotimmunoassay for monocional and other antibodies. Anal Biochem 119, 142-147

Kilshaw PJ, Sissons JW (1979) Gastrointestinal allergy to soyabean protein in preruminant calves. Antibody production and digestive 
disturbances in calves fed heated soyabean flour. Res Vet Sci 27, 361-365

Maga JA (1981) Measurement of available Iysine using the guanidination reaction. $J$ Food Sci $46,132-134$

Pedersen HE, Sissons JW (1984) Effect of antigenic soyabean protein on the physiology and morphology of the gut in the preruminant calf. Can J Anim Sci 64 (Suppl), s183-s184

Richterich R, Colombo JP (1978) In: Klinische Chemie (Richterich R, Colombo JP, eds) Karger AG, Bâle, 391-392

Rollin RE, Mero KD, Kosizek PD, Philipps RW (1986) Diarrhea and malabsorption in calves associated with therapeutic doses of antibiotics: Absorptive and clinical changes. Am $J$ Vet Res 47, 987-991

Scharrer $E$ (1978) Concentrative amino acid uptake at the serosal side of colon mucosa. Pflügers Arch, Eur J Physiol 376, 245-249

Seegraber FJ, Morrill JL (1979) Effect of soy protein on intestinal absorptive ability of calves by the xylose absorption test. J Dairy Sci $62,972-977$

Sissons JW, Smith RH (1976) The effect of different diets including those containing soyabean products, on digesta movement and water and nitrogen absorption in the small in- testine of the pre-ruminant calt. Br J Nutr 36 , 421-438

Ternouth JH, Roy JHB, Siddons RC (1974) Concurrent studies of the flow of digesta in the duodenum and of exocrine pancreatic secretion of calves. Br J Nutr 31, 13-26

Tzeng D, Davis CL (1980) Amino acid nutrition of the young calf. Estimation of methionine and lysine requirements. J Dairy Sci 63, 441450

Wilson TH, Wiseman G (1954) The use of sacs of everted small intestine for the study of the transference of substances from the mucosal to the serosal surface. J Physiol London 123 , 116-124

Woode GN, Smith C, Dennis MJ (1978) Intestinal damage in rotavirus infected calves assessed by D-xylose malabsorption. Vet Rec 102, 340-341

Young VR, Alexis SD, Baliga BS, Munro HN, Muecke W (1972) Metabolism of administered 3-methylhistidine. Lack of muscle transfer ribonucleic acid charging and quantitative excretion as 3-methylhistidine and its $\mathrm{N}$-acetyl derivative. J Biol Chem 247, 3592-3600

Yvon M, Pelissier JP, Guilloteau P, Toullec R (1986) Digestion des protéines du lait dans la caillette du veau pré-ruminant. Reprod Nutr Dev 26, 705-715 